\title{
MORE ON THE SCHUR SUBGROUP
}

\author{
MURRAY M. SCHACHER ${ }^{1}$
}

ABSTRACT. Let $k$ be an abelian extension of the rational field $Q$. We show Schur's subgroup $S(k)$ of the Bauer group $B(k)$ is usually of infinite index. Generators for $p$-torsion elements of $S(k)$ are found when $k$ is the cyclotomic field of $p$ th roots of unity.

Let $k$ be an algebraic number field. We write $B(k)$ for the Brauer group of $k$, and $B_{n}(k)$ for the subgroup of $B(k)$ generated by classes of division rings of exponent $n$. Let $S(k)$ be the subgroup of $B(k)$ consisting of all classes which contain a simple component of $Q[G]$, the group algebra of a finite group $G$ over the rational field $Q$. Following [6] we call $S(k)$ the Schur subgroup of $k$. Let $S_{n}(k)=S(k) \cap B_{n}(k)$. In [5] the structure of $S_{3}(k)$ for $k=Q(\sqrt{ }(-3))$ is determined. Theorem 2 of this note generalizes the results of [5].

If $A$ is a central simple algebra over $k$, we write [ $A]$ for the corresponding class in $B(k)$.

THEOREM 1. $S_{n}(k)$ is of infinite index in $B_{n}(k)$ for all $n \geqq 2$ unless $n=2$ and $k=Q$. In the exceptional case $B_{2}(Q)=S_{2}(Q)$.

Proof. Since $S(k)$ is trivial unless $k$ is the field of an irreducible character of a finite group $G$, we may assume $k / Q$ is abelian. Assume $k \neq Q$ and $r=[k: Q]$. There are infinitely many primes of $Q$ which split completely in $k$; let $p_{1}, \cdots, p_{n}, \cdots$ be an infinite list of them. For each $p_{i}$ let $\mathrm{g}_{i}^{1}, \cdots, \mathfrak{g}_{i}^{r}$ be the primes of $k$ lying over $p_{i}$. We construct $\left[D_{1}\right],\left[D_{2}\right], \cdots$, $\left[D_{m}\right], \cdots$ in $B_{n}(k)$ as follows:

$D_{m}$ is the central division ring over $k$ whose Hasse invariants satisfy:

$$
\begin{aligned}
\operatorname{inv}_{\mathfrak{g}_{2 m-1}^{1}} D_{m} & =\frac{1}{n}, \quad \operatorname{inv}_{\mathfrak{g}_{2 m}^{1}} D_{m}=-\frac{1}{n}, \\
\quad \operatorname{inv}_{\mathfrak{g}} D_{m} & =0 \quad \text { at all other primes } \mathfrak{g} \text { of } k .
\end{aligned}
$$

The construction of the $D_{m}$ is allowed by [1, Theorem 7.8]. By [2] we

Received by the editors January 29, 1971.

AMS 1970 subject classifications. Primary 20C05; Secondary 12B10.

Key words and phrases. Brauer group, cyclotomic, exponent, group algebra, Hasse invariant, index, prime, Schur subgroup, tamely ramified, unramified.

${ }^{1}$ The preparation of this paper was supported in part by NSF Grant No. GP-23107. 
have: $[D] \in S_{n}(k) \Rightarrow$ for each $i, D$ has constant index at $\mathrm{g}_{i}^{1}, \cdots, \mathrm{g}_{i}^{r}$. Hence $\left[D_{1}\right], \cdots,\left[D_{m}\right], \cdots$ above represent distinct cosets of $S_{n}(k)$ in $B_{n}(k)$. It follows that $\left[B_{n}(k): S_{n}(k)\right]=\infty$.

If $k=Q$, then $S(k)=S_{2}(k)$ by the Brauer-Speiser theorem (see [6]). The fact that $B_{2}(Q)=S_{2}(Q)$ follows from [4].

Theorem 1 was also noted by Burton Fein.

Let $p$ be a fixed odd prime. We will classify the algebras of index $p$ in $S(k)$, where $k=Q\left(\xi_{p}\right)$ is the cyclotomic field of $p$ th roots of unity. This generalizes Theorem 2 of [5].

If $q$ is a prime, $q \equiv 1(\bmod p)$, then $q$ splits completely in $k=Q\left(\xi_{p}\right)$. Let $\mathrm{g}_{1}, \cdots, \mathrm{g}_{p-1}$ be the primes of $k$ lying over $q$. The field $L=Q\left(\xi_{q}, \xi_{p}\right)$ is cyclic over $Q\left(\xi_{p}\right)$ of degree $q-1$; let $\tau$ be the generator of the Galois group of $L$ over $k$. Let $H$ be the group generated by $x, y$, and $z$ where $x^{p}=y^{q}=1, z$ acts on $\langle y\rangle$ according to the Galois action of $\tau$ on $Q\left(\xi_{q}\right)$, $z^{q-1}=x$, and $x$ is central in $H$. Then the cyclic algebra $\mathfrak{A}=\left(k\left(\xi_{q}\right), \tau, \xi_{p}\right)$ is a homomorphic image of $Q[H]$, so [भ] is in the Schur subgroup of $k$. Clearly $\mathfrak{A}^{p}$ is a total matrix algebra since $\xi_{p}^{p}=1$; so [थ'] has order 1 or $p$ in $B(k)$. [थ] has order $1 \Leftrightarrow \xi_{p}$ is a norm from $L=k\left(\xi_{q}\right)$ to $k$. We show $\xi_{p}$ is not a local norm at the primes $\mathrm{g}_{1}, \cdots, \mathfrak{g}_{p-1}$ above. For convenience we fix $\mathfrak{g}=\mathfrak{g}_{1}$.

The extension $L / k$ is totally and tamely ramified at $\mathrm{g}$; let $t$ be the unique prime of $L$ lying over g. If $U_{t}$ (resp. $U_{\mathrm{g}}$ ) denotes the units of $L_{t}$ (resp. $k_{\mathrm{g}}$ ) and $U_{t}^{1}\left(\operatorname{resp} . U_{\mathfrak{g}}^{1}\right)$ those which are $1(\bmod t)(\operatorname{resp} .1(\bmod g))$, then as in $[7, v, \# 3]$ the norm induces a homomorphism:

$$
N_{0}: U_{t} / U_{t}^{1} \rightarrow U_{\mathfrak{g}} / U_{\mathrm{g}}^{1} .
$$

But $U_{t} / U_{t}^{1} \cong L_{t}^{*}$, the multiplicative group of the residue class field of $L$ at $t$. Similarly $U_{\mathfrak{g}} / U_{\mathfrak{g}}^{1} \cong \bar{k}_{\mathfrak{g}}^{*} \cong \bar{L}_{t}^{*} \cong Z_{q}^{*}$ as $\mathfrak{g}$ is totally ramified in $L$. Thus (1) reduces to a homomorphism:

$$
N_{0}: Z_{q}^{*} \rightarrow Z_{q}^{*}
$$

of cyclic groups of order $q-1$. By [7, Proposition 5, p. 92] we have: $N_{0}(x)=x^{q-1}$ in (2). Hence the image of $N_{0}$ is trivial, so $N_{0}$ does not cover the image of $\xi_{p}$; it follows that $\xi_{p}$ is not a norm.

Thus [ㅁ] represents an element of order $p$ in $S(k)$. Clearly $\mathfrak{A}$ is split at all primes $w, w \notin\left\{\mathfrak{g}_{1}, \cdots, \mathfrak{g}_{p-1}\right\}$, for each such prime is unramified from $k$ to $L$ and so $\xi_{p}$ is a unit, hence a norm, at $w$. By the proof of Theorem 5 of [3] we have with suitable relabelling, invariants of [भI] of form $1 / p$, $2 / p, \cdots,(p-1) / p$ at $\mathfrak{g}_{1}, \cdots, \mathfrak{g}_{p-1}$.

We claim $S_{p}(k)$ is generated by the classes [थ] above. Suppose $D$ is a central division algebra over $k$ with $[D] \in S_{p}(k) ; D$ has exponent $p . b$ If is a rational prime, $q \equiv 1(\bmod p)$, and $g_{1}, \cdots, g_{p-1}$ are the primes of 
$k=Q\left(\xi_{p}\right)$ lying over $q$, we have by [2], $\operatorname{inv}_{\mathrm{g}_{1}} D=0 \Rightarrow \operatorname{inv}_{\mathbf{g}_{i}} D=0, i=1$, $\cdots, p-1$. Assume $\operatorname{inv}_{\mathrm{g}_{1}} D=a / p,(a, p)=1$. Set $x=[\mathfrak{U}]^{-a} \cdot[D] \in$ $S_{p}(k)$; then $x$ has invariant 0 at $\mathrm{g}_{1} \Rightarrow \operatorname{inv}_{\mathrm{g}_{i}} x=0$ for $i=1, \cdots, p-1 \Rightarrow$ $\operatorname{inv}_{\mathfrak{g}_{i}} D=a i / p=i(a / p)$. Thus $D$ has invariants of type $1 / p, 2 / p, \cdots$, $(p-1) / p$ at split primes over $q$ where $q \equiv 1(\bmod p)$. We must show that $D$ has no other non-0 invariants. By appropriate multiplication in $B(k)$ as above we may assume $\operatorname{inv}_{\mathfrak{g}} D=0$ for all $q$ lying over completely split primes of $Q$.

$D$ has no non-0 invariants at primes of $k$ lying over odd rational primes by [8, Satz 10]. Also, since the index of $D$ is $p$ and $p \neq 2$, then $D$ has no non- 0 invariants at primes of $k$ extending 2 by [8, Satz 11]. We have proved:

THEOREM 2. If $p$ is an odd prime, then the division rings $D$ with $[D] \in$ $S_{p}(k), k=Q\left(\xi_{p}\right)$ have invariants of type $1 / p, 2 / p, \cdots,(p-1) / p$ at completely split primes of $k$, and 0 everywhere else. The classes [भ] above generate $S_{p}(k)$.

We note that Theorem 2 has the following unusual consequence: If $p$ and $q$ are distinct odd primes, then $\xi_{p}$ is not a norm from $Q\left(\xi_{p}, \xi_{q}\right)$ to $Q\left(\xi_{p}\right) \Leftrightarrow q \equiv 1(\bmod p)$.

Many thanks are due to both Burton Fein and Basil Gordon for pointing out to me the existence and applicability of [8]. Ken Fields has noted that there is no way of determining $S_{p^{2}}\left(Q\left(\xi_{p^{2}}\right)\right)$ without first determining $S_{p}\left(Q\left(\xi_{p^{2}}\right)\right)$.

\section{REFERENCES}

1. E. Artin and J. Tate, Class field theory, Harvard University, Cambridge, Mass., 1961.

2. M. Benard, The Schur subgroup. I, J. Algebra (to appear).

3. B. Fein and M. Schacher, Embedding finite groups in rational division algebras. I, J. Algebra 17 (1971), 412-428. 223.

4. K. L. Fields, On the Brauer-Speiser theorem, Bull. Amer. Math. Soc. 77 (1971),

5. - On the Schur subgroup, Bull. Amer. Math. Soc. 77 (1971), 477-478.

6. K. L. Fields and I. N. Herstein, On the Schur subgroup of the Brauer group, J. Algebra (to appear).

7. J.-P. Serre, Corps locaux, Publ. Inst. Math. Univ. Nancago, VIII, Actualités Sci. Indust., no. 1296, Hermann, Paris, 1962. MR 27 \#133.

8. E. Witt, Die Algebraische Struktur des Gruppenringes einer endlichen Gruppe über einem Zahlkörper, J. Reine Angew. Math. 190 (1952), 231-245. MR 14, 845.

Department of Mathematics, University of California, Los Angeles, CaliFORNIA 90024 